\title{
Universal properties of penetrative turbulent Rayleigh-Bénard convection
}

\author{
Qi Wang $\odot,{ }^{1,2}$ Philipp Reiter, ${ }^{3}$ Detlef Lohse $\odot,{ }^{1,3, *}$ and Olga Shishkina ${ }^{3, \dagger}$ \\ ${ }^{1}$ Physics of Fluids Group and Max Planck Center for Complex Fluid Dynamics, \\ MESA+ Institute and J. M. Burgers Centre for Fluid Dynamics, \\ University of Twente, P.O. Box 217, 7500AE Enschede, The Netherlands \\ ${ }^{2}$ Department of Modern Mechanics, University of Science \\ and Technology of China, Hefei 230027, China \\ ${ }^{3}$ Max Planck Institute for Dynamics and Self-Organization, 37077 Göttingen, Germany
}

(Received 15 December 2020; accepted 7 June 2021; published 21 June 2021)

\begin{abstract}
Penetrative turbulence, which occurs in a convectively unstable fluid layer and penetrates into an adjacent, originally stably stratified layer, is numerically and theoretically analyzed. As example we pick the canonical Rayleigh-Bénard geometry, but now with the bottom plate temperature $T_{b}>4{ }^{\circ} \mathrm{C}$, the top plate temperature $T_{t} \leqslant 4^{\circ} \mathrm{C}$, and the density maximum around $T_{m} \approx 4^{\circ} \mathrm{C}$ in between, resulting in penetrative turbulence. Next to the Rayleigh number Ra, the crucial new control parameter as compared to standard Rayleigh-Bénard convection is the density inversion parameter $\theta_{m} \equiv\left(T_{m}-T_{t}\right) /\left(T_{b}-T_{t}\right)$. The crucial response parameters are the relative mean midheight temperature $\theta_{c}$ and the overall heat transfer (i.e., the Nusselt number $\mathrm{Nu}$ ). We numerically show (for Ra up to $10^{10}$ ) and theoretically derive that $\theta_{c}\left(\theta_{m}\right)$ and $\mathrm{Nu}\left(\theta_{m}\right) / \mathrm{Nu}(0)$ are universally (i.e., independently of Ra) determined only by the density inversion parameter $\theta_{m}$ and succeed to derive these universal dependences. In particular, $\theta_{c}\left(\theta_{m}\right)=\left(1+\theta_{m}^{2}\right) / 2$, which holds for $\theta_{m}$ below a Ra-dependent critical value, beyond which $\theta_{c}\left(\theta_{m}\right)$ sharply decreases and drops down to $\theta_{c}=1 / 2$ at $\theta_{m}=\theta_{m, c}$. This critical density inversion parameter $\theta_{m, c}$ can be precisely predicted by a linear stability analysis. Finally, we numerically identify and discuss rare transitions between different turbulent flow states for large $\theta_{m}$.
\end{abstract}

DOI: 10.1103/PhysRevFluids.6.063502

\section{INTRODUCTION}

Turbulent Rayleigh-Bénard convection (RBC) [1-3], which occurs in a fluid layer due to a temperature difference at its bottom and top surfaces, is the paradigmatic model system to study thermally driven turbulence. Usually it is considered within the Oberbeck-Boussinesq (OB) approximation, in which all fluid properties are assumed to be constant, apart from the density in the buoyancy term of the momentum equation, where it is assumed to be linearly dependent on the temperature. However, in many cases in nature and technology, the density of many fluids is strongly nonlinear and even nonmonotonic with the temperature, which significantly influences the flow patterns and the heat transport properties in the system. The most famous and relevant example is water, for which the density is maximal at $T_{m} \approx 4{ }^{\circ} \mathrm{C}$. This density maximum has a dramatic and pronounced influence on many natural phenomena like the freezing of lakes and estuaries, and the survival of fauna in shallow waters in winter [4-7], as they do not freeze from the bottom to

*d.lohse@utwente.nl

†Olga.Shishkina@ds.mpg.de 
the top. It also strongly affects the melting of ice in water. However, also here RBC can be used again as paradigmatic model system, namely, when $T_{m}$ is in between the top and bottom plate temperature. This is an example of so-called penetrative convection $[8,9]$, where convection in a thermally unstable layer penetrates into the adjacent stable layers. Penetrative convection is not only important for water around $4{ }^{\circ} \mathrm{C}$ and freezing lakes, etc., but also in many astrophysical settings, for example, in the tachocline of the Sun [10] and possibly in the liquid core of the Earth [11] and in the Jupiter's atmosphere [12].

The control parameters in this problem are the Prandtl number $\operatorname{Pr} \equiv \nu / \kappa$, where $v$ is the kinematic viscosity and $\kappa$ the thermal diffusivity of the fluid, the aspect ratio $\Gamma \equiv W / H$ as ratio of the width $W$ and the height $H$ of the domain, and the Rayleigh number Ra $\equiv g \alpha \Delta^{q} H^{3} / v \kappa$, where $H$ is the height of the cell and $g$ the gravitational acceleration. Note that the nonlinear dependence of Ra on the temperature difference $\Delta \equiv T_{b}-T_{t}$ between the hot bottom plate temperate $T_{b}>T_{m}$ and the cold top plate temperature $T_{t} \leqslant T_{m}$ reflects the nonlinear relationship between the density and the temperature around the density maximum, namely, $\rho=\rho_{m}\left(1-\alpha\left|T-T_{m}\right|^{q}\right)$ [13], where $\rho_{m} \approx 1000 \mathrm{~kg} / \mathrm{m}^{3}$ is the maximum density at the temperature $T_{m} \approx 4{ }^{\circ} \mathrm{C}$. Here we take the measured material properties for water, namely $q=1.895$ and $\alpha=9.30 \times 10^{-6}(\mathrm{~K})^{-q}$ as isobaric thermal expansion coefficient. Apart from the density, all other material properties of the fluid are assumed to be constant. The crucial new control parameter in penetrative convection as compared to standard RBC is the density inversion parameter $\theta_{m} \equiv\left(T_{m}-T_{t}\right) / \Delta$. We consider $0 \leqslant \theta_{m}<1$, with the limiting case $\theta_{m}=0$ (or $T_{t}=T_{m}$ ) without a stably stratified region and thus being closest to standard RBC and the other limiting case $\theta_{m}=1$ (or $T_{b}=T_{m}$ ) being fully stably stratified.

The response parameters of the system are the Nusselt number $\mathrm{Nu} \equiv Q H /(k \Delta)$ and the Reynolds number $\mathrm{Re} \equiv U H / \nu$, which indicate the nondimensional heat transport and flow strength in the system, respectively. Here $Q$ is the heat flux crossing the system, $k$ the thermal conductivity, and $U \equiv\left\langle\boldsymbol{u}^{2}\right\rangle_{V, t}^{1 / 2}$ the time and volume averaged root-mean-square velocity of the flow. The central new response parameter in penetrative convection as compared to standard RBC is the mean temperature $T_{c}$ at midheight, or, when nondimensionalized, $\theta_{c}=\left(T_{c}-T_{t}\right) / \Delta$. Whereas in standard RBC $\theta_{c}=$ $1 / 2$ for symmetry reasons, here in penetrative convection $1 / 2 \leqslant \theta_{c}<1$, reflecting that the hot fluid from the bottom unstably stratified region can more easily reach the center than the cold fluid from top stably stratified region.

Early studies of penetrative RBC focused on the stability of the flows near the onset of convection [8,9,14-17]. Recently, turbulent penetrative RBC attracted significant attention. These studies considered either cold water near $4{ }^{\circ} \mathrm{C}[7,18-20]$ or other fluids with a density maximum at certain conditions [21-27]. Much attention has been paid to mixing, the generation of internal waves, mean flows, global heat and momentum transport, and so on. Reference [18] studied the internal gravity wave excitation by convection of cold water near $4{ }^{\circ} \mathrm{C}$ using two-dimensional (2D) direct numerical simulations (DNS). Ref. [22] found that oscillating mean flow spontaneously develops from turbulently generated internal waves. Reference [7] studied coupling of icing and turbulent convection of cold water near $4{ }^{\circ} \mathrm{C}$, and found that the density maximum effect can drastically change system behaviors. Very recently, Refs. [26,27] studied penetrative convection in the background of subglacial lakes and found that dynamic flows create potentially habitable conditions in Antarctic subglacial lakes.

Global heat and momentum in penetrative $\mathrm{RBC}$ of cold water near $4{ }^{\circ} \mathrm{C}$ were studied in Ref. [19]. It was shown that for $0 \leqslant \theta_{m} \lesssim 0.9$, the mean central temperature $\theta_{c}$, the normalized Nusselt number $\operatorname{Nu}\left(\theta_{m}\right) / \mathrm{Nu}(0)$, and the normalized Reynolds number $\operatorname{Re}(\theta) / \operatorname{Re}(0)$ are almost independent of $\mathrm{Ra}$ and thus to be claimed to be universal. However, in this study we will show that when extending the range of the control parameter $\theta_{m}$ towards 1 (i.e., towards the fully stably stratified case), the center temperature $\theta_{c}$ strongly depends on Ra. Moreover, we propose a model for the dependence of this key response parameter $\theta_{c}$ on the density inversion parameter $\theta_{m}$ and demonstrate its excellent agreement with the DNS data. Also the dependence of the other central response parameter, namely, the Nusselt number, on $\theta_{m}$ is theoretically explained. 


\section{SIMULATION DETAILS}

The dimensionless governing equations are the following:

$$
\begin{aligned}
\nabla \cdot \boldsymbol{u} & =0, \\
\partial \boldsymbol{u} / \partial t+\boldsymbol{u} \cdot \nabla \boldsymbol{u} & =\sqrt{\operatorname{Pr} / \operatorname{Ra}} \nabla^{2} \boldsymbol{u}-\nabla p+\left|\theta-\theta_{m}\right|^{q} \overrightarrow{\boldsymbol{e}}_{z}, \\
\partial \theta / \partial t+\boldsymbol{u} \cdot \nabla \theta & =1 / \sqrt{\operatorname{Ra} \operatorname{Pr}} \nabla^{2} \theta
\end{aligned}
$$

where $\boldsymbol{u}=(u, w), \theta$, and $p$ are the velocity, temperature, and pressure, respectively. For nondimensionalization, we choose $H$ and $U_{f}=\left(g \alpha \Delta^{q} H\right)^{1 / 2}$ as the reference length and velocity. The reference time is free-fall time $t_{f}=H / U_{f}$. Temperature is nondimensionalized as $\theta=\left(T-T_{t}\right) / \Delta$. We consider periodic boundary conditions (BCs) in the horizontal direction and no-slip isothermal $\mathrm{BCs}$ at the top and bottom boundaries.

We perform 2D DNS in a broad Ra range, $10^{7} \leqslant \mathrm{Ra} \leqslant 10^{10}$, with fixed aspect ratio $\Gamma=2$. $\mathrm{Pr}$ is fixed to 11.57 in all simulations, which corresponds to the value for water at $4{ }^{\circ} \mathrm{C}$. In addition, we perform $3 \mathrm{D}$ DNS for $\mathrm{Ra}=10^{7}$ and $\Gamma=4$. The governing equations are solved with the secondorder finite-difference code AFiD [28,29], which has already been extensively used to study RBC; see, e.g., Refs. [30,31]. The used staggered grids satisfy the resolution requirements for DNS [32]. Details on the simulations can be found in Appendix A.

\section{RESULTS}

Figure 1 gives an idea of the flow organization. Figures 1(a)-1(c) show 2D instantaneous temperature fields for different $\theta_{m}$ and $\mathrm{Ra}=10^{10}$. For $\theta_{m}>0$, only the lower layer in the cell can be convectively unstable. For not too large $\theta_{m}$, convection penetrates from the lower layer into the upper, gravitationally stable, layer, and this increases the bulk temperature. For $\theta_{m}=0.5$ [Fig. 1(a)], the convective flow occupies almost the whole domain, while for $\theta_{m}=0.9$ [Fig. 1(b)] a stably stratified layer forms near the top plate. For even larger $\theta_{m}=0.965$ [Fig. 1(c)], the flow stratification takes place nearly in the whole cell. A similar change of the global flow structure with increasing $\theta_{m}$ is observed in the 3D DNS [Figs. 1(d)-1(f)]. For the penetrative convection with $\theta_{m}>0$, an interface between the stratified and convective regions is identified, below which exists vigorous turbulent convection, and above which the flow is stably stratified and generates internal waves $[18,20,22,23]$.

The height at which time- and horizontally averaged temperature reaches $4{ }^{\circ} \mathrm{C}$ is displayed in Fig. 2. Again, it is seen that for relatively small $\theta_{m} \lesssim 0.5$, the convection region occupies almost the whole domain. In contrast, for large $\theta_{m} \gtrsim 0.5$, the area of the convective region significantly decreases with increasing $\theta_{m}$. Up to a critical $\theta_{m}=\theta_{m, c}$, the flow is in a conduction state without penetrative convection, and therefore $H_{m} / H=1-\theta_{m}$.

The intrusion of penetrating convection from the convectively unstable regions to convectively stable ones resembles those observed in internally heated convection [33-35] and horizontal convection [36-38], as well as in counterrotating Taylor-Couette flows, where stratified angular velocity zones are located near the outer cylinder [39-41].

The time- and area-averaged temperature profiles and their dependences on $\theta_{m}$ for $\mathrm{Ra}=10^{10}$ are presented in Fig. 3(a). It shows that for $\theta_{m}=0$, the profile is similar to that in the OB case, with the mean central temperature $\theta_{c}$ being close to the arithmetic mean of the top and bottom temperatures $\left(\theta_{c}=1 / 2\right)$. One can see that with increasing $\theta_{m}$, the temperature in the thermally unstable, lower zone gradually increases. However, the mean temperature $\theta_{c}$ at midheight behaves nonmonotonically. With increasing $\theta_{m}$, it first increases as long as the half height location $(z / H=$ 0.5 ) lies in the thermally unstable region, but then it starts to decrease back to the arithmetic mean of the top and bottom temperatures, once the midheight location is swallowed up by the upper stably stratified region. 
(a)

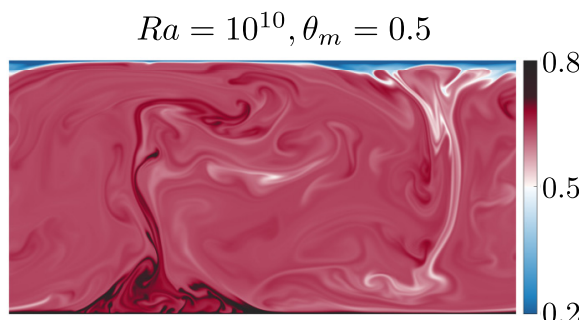

(b)

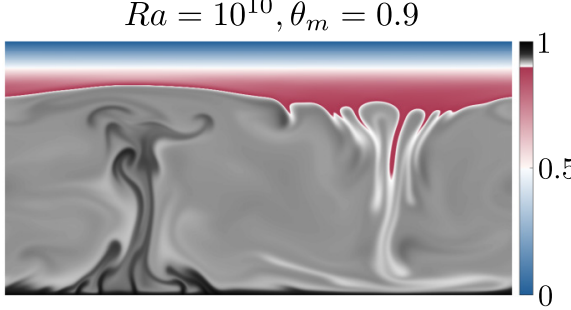

(c)

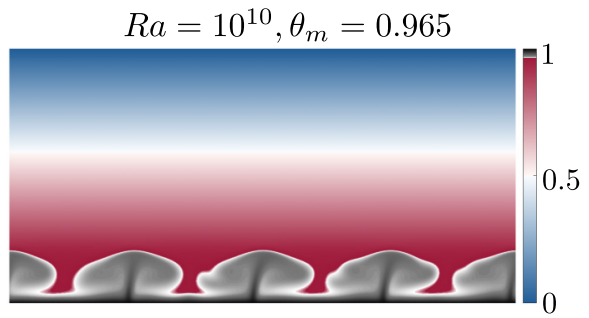

(d)

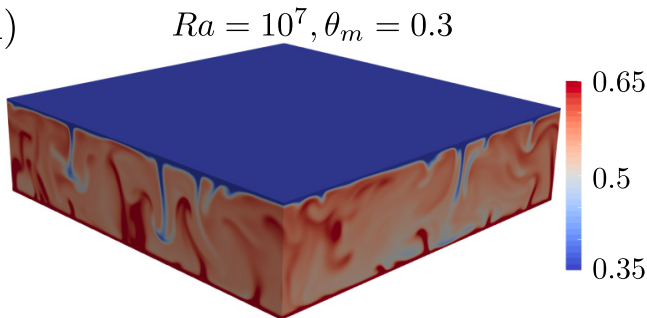

(e)

$$
R a=10^{7}, \theta_{m}=0.7
$$

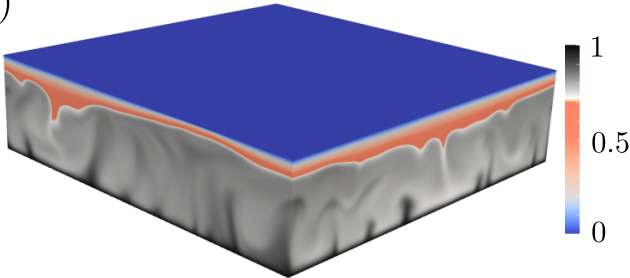

(f)

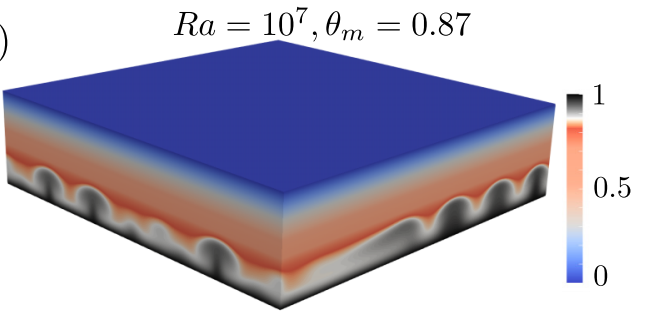

FIG. 1. Instantaneous temperature fields for different $\theta_{m}$ in (a-c) 2D DNS for Ra $=10^{10}$ and $\Gamma=2$ and (d-f) 3D DNS for Ra $=10^{7}$ and $\Gamma=4$ : (a) $\theta_{m}=0.5$, (b) $\theta_{m}=0.9$, (c) $\theta_{m}=0.965$, (d) $\theta_{m}=0.3$, (e) $\theta_{m}=0.7$, and (f) $\theta_{m}=0.87$.

Figure 3(b) shows the mean central temperature $\theta_{c}$ as function of $\theta_{m}$, for both the 2D and the 3D cases. We note that the collapse of the 3D data with the 2D data confirms the similarity between 2D and 3D RBC for large Pr [42]. One can observe two different regimes: in regime I, for low-density

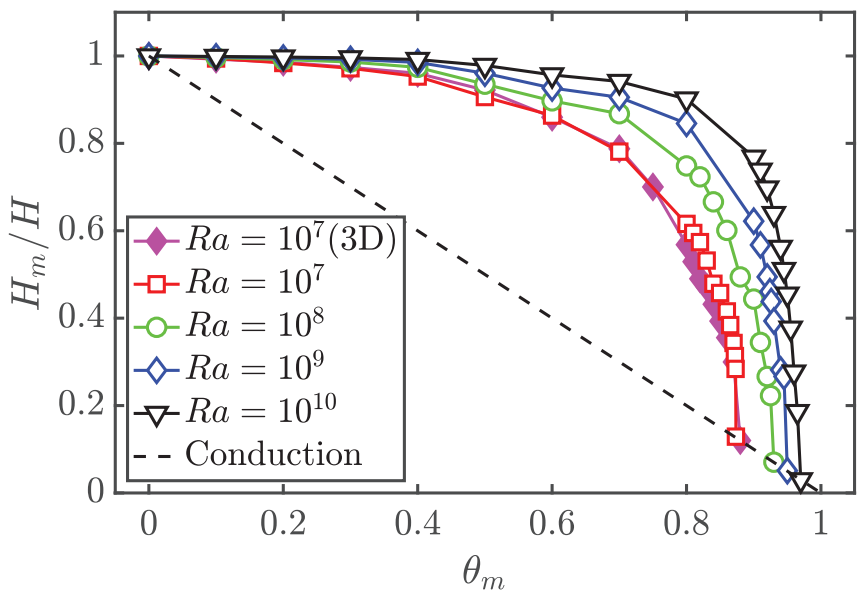

FIG. 2. The dimensionless height $H_{m} / H$ at which the time- and horizontally averaged temperature is $4{ }^{\circ} \mathrm{C}$, namely, $\theta=\theta_{m}$. The dashed line denotes $H_{m} / H$ when the flow is in a conduction state without penetrative convection. 

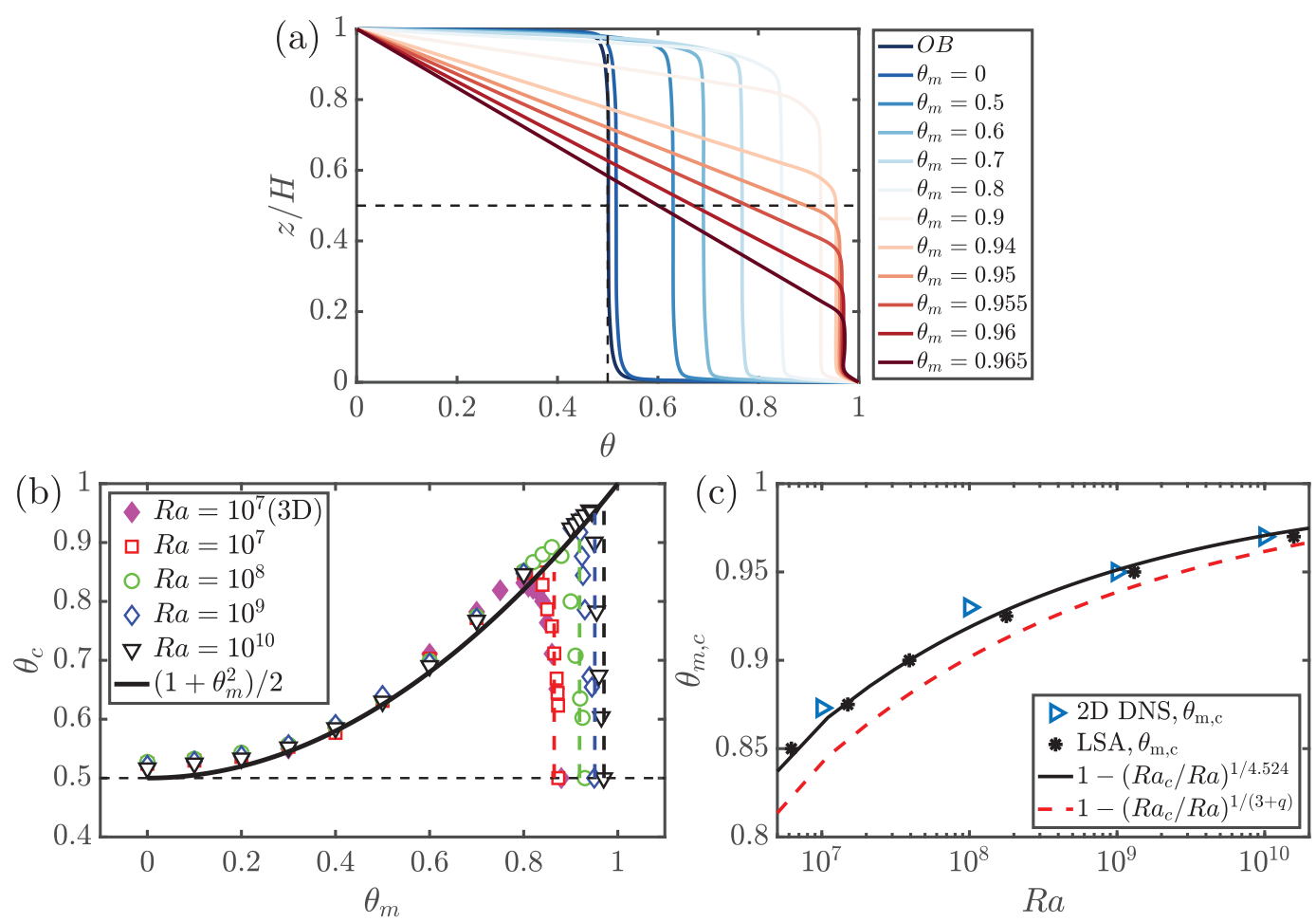

FIG. 3. (a) Time- and horizontally averaged temperature profiles for different $\theta_{m}$, as obtained in the 2D DNS for $\mathrm{Ra}=10^{10}$ and $\Gamma=2$. (b) Time- and horizontally averaged temperature at midheight, $\theta_{c}$, as function of $\theta_{m}$, in the 2D (open symbols) and 3D (closed symbols) cases for different Ra. The solid line shows the theoretical prediction $\theta_{c}=\left(1+\theta_{m}^{2}\right) / 2$, which is universal for all Ra. Predictions of the linear stability analysis for the critical $\theta_{m}=\theta_{m, c}$, where $\theta_{c}$ drops back to $1 / 2$, depend on Ra and are shown with the dashed vertical lines of the colors that correspond to the DNS data. (c) The critical $\theta_{m, c}$ versus Ra, as obtained from the 2D DNS (open symbols), linear stability analysis (closed symbols and their fit shown with the solid line), and the theoretical model $\theta_{m, c}=1-\left(\mathrm{Ra}_{c} / \mathrm{Ra}\right)^{1 /(3+q)}$, with $\mathrm{Ra}_{c}=1171$ and $q=1.895$ (dashed line).

inversion parameter $0 \leqslant \theta_{m} \ll 1$, the central temperature $\theta_{c}$ monotonically increases with increasing $\theta_{m}$, and all the data collapse onto a single curve, independently of Ra. However, in regime II, for $\theta_{m}$ close to 1 , with increasing $\theta_{m}$ the central temperature $\theta_{c}$ extremely sharply drops to the value $1 / 2$. We denote the value of $\theta_{m}$, at which $\theta_{c}$ reaches $1 / 2$ in regime II, as $\theta_{m, c}$, i.e., $\theta_{c}\left(\theta=\theta_{m, c}\right)=1 / 2$. It is clear that $\theta_{m, c}$ increases with increasing Ra and approaches 1 for $\mathrm{Ra} \rightarrow \infty$, implying that for strong enough thermal driving the whole cell is filled with penetrative turbulence, even for large density inversion parameter close to 1 .

We now set out to theoretically explain the universal dependence of the central temperature $\theta_{c}$ on the control parameter $\theta_{m}$ in regime I. First, we notice that for $\theta_{m}=0$, the temperature of the fluid is larger than $T_{m}$ through the entire convection cell and therefore the situation is similar to the OB case. Thus, the central temperature can be well approximated by the arithmetic mean of the top and bottom temperatures, i.e., $\theta_{c}=1 / 2$. With increasing $\theta_{m}$, the height, at which the time- and area-averaged temperature equals $T_{m}$, gradually decreases from the top towards the bottom, and at a certain moment arrives at the midheight, which indicates the end of regime I. Therefore, when $\theta_{m} \rightarrow$ 1 within regime I, the central temperature $\theta_{c}$ tends to $\theta_{m}$ and $\lim _{\theta_{m} \rightarrow 1} \frac{1-\theta_{c}}{1-\theta_{m}}=1$. Applying L'Hôpital's rule to this limit, we obtain that $\lim _{\theta_{m} \rightarrow 1} \frac{d \theta_{c}}{d \theta_{m}}=1$. Thus, for $\theta_{m} \rightarrow 1$, one can take $\theta_{c}=\theta_{m}$ and $d \theta_{c} / d \theta_{m}=1$. This together with $\theta_{c}=1 / 2$ at $\theta_{m}=0$ gives a simple polynomial approximation of 
the mean central temperature within the regime I,

$$
\theta_{c}=\left(1+\theta_{m}^{2}\right) / 2 \text {. }
$$

Figure 3(b) demonstrates that the model (4) accurately represents the 2D and 3D DNS data within regime $\mathrm{I}$.

Next we will explain the abrupt drop of the central temperature near $\theta_{m, c}$ as function of Ra. Therefore, for any given Ra, we must find $\theta_{m}=\theta_{m, c}$, at which the central temperature $\theta_{c}$ drops back to $1 / 2$ in regime II; see Fig. 3(b). As discussed above, the lower layer of the cell is convectively unstable for small $\theta_{m}$. However, the situation changes dramatically for larger $\theta_{m}$, when the Rayleigh number $\mathrm{Ra}_{\ell}$ associated with this layer becomes as small as the critical Rayleigh number $\mathrm{Ra}_{c}$ for the onset of convection in a domain with no-slip BCs at its lower surface and free-slip BCs at its top surface, at which the temperature is kept equal to $T_{m}$. When at a certain $\theta_{m}=\theta_{m, c}$ the value of Ra $\ell_{\ell}$ becomes equal to $\mathrm{Ra}_{c}$, the lower layer becomes convectively stable and stratified as the upper layer, and therefore the central temperature in the whole cell becomes equal to the arithmetic mean of the top and bottom temperatures of the cell, i.e., $\theta_{c}=1 / 2$.

The Rayleigh number $\mathrm{Ra}_{\ell}$ associated with such stratified lower layer is related to $\mathrm{Ra}$ as $\mathrm{Ra}_{\ell}=$ $\left(1-\theta_{m, c}\right)^{3+q} \mathrm{Ra}$. This is due to the reduction of the height of the layer to $\left(1-\theta_{m, c}\right) H$, compared to the height $H$ of the whole cell, and due to the reduction of the temperature difference between the boundaries of the layer to $\left(1-\theta_{m, c}\right) \Delta$, compared to the temperature difference $\Delta$ at the cell plates. Equating $\mathrm{Ra}_{\ell}=\mathrm{Ra}_{c}$, we obtain the prediction

$$
\theta_{m, c}=1-\left(\mathrm{Ra}_{c} / \mathrm{Ra}\right)^{1 /(3+q)} .
$$

The critical $\mathrm{Ra}_{c}$ for the onset of convection in an infinite layer of cold water with the top temperature $T_{m}$, no-slip BCs at the bottom and free-slip BCs at the top equals $\mathrm{Ra}_{c}=1171$, as we calculated using linear stability analysis (LSA); see also Appendix B for more information. The results produced by the model (5) are plotted as dashed line in Fig. 3(c). The model prediction for the behavior of $\theta_{m, c}$ as function of Ra is consistent with the DNS results (open symbols).

Even more accurate predictions of $\theta_{m, c}$ can be obtained with the LSA applied to a 2D convection cell filled with cold water, for the cell aspect ratio $\Gamma=2$, and periodic BC at the side walls. Thus, for any given $\theta_{m}=\theta_{m, c}$, the LSA provides the critical Rayleigh number Ra for the onset of convection; a relation between them is precisely described by $\theta_{m, c}=1-(1171 / \mathrm{Ra})^{1 / 4.524}$; see Appendix B. These predictions by the LSA are in a good agreement with the DNS data, as can be seen in Fig. 3(c).

We now focus on how the Nusselt number $\mathrm{Nu}$ depends on the control parameter $\theta_{m}$ and develop a model for the reduced Nusselt number, $\mathrm{Nu}\left(\theta_{m}\right) / \mathrm{Nu}\left(\theta_{m}=0\right)$, based on our knowledge on the heat transport properties in the OB case. Figure 4(a) shows that for all Ra, the absolute values of $\mathrm{Nu}\left(\theta_{m}\right)$ monotonically decrease with increasing $\theta_{m}$. After normalization using $\mathrm{Nu}\left(\theta_{m}=0\right)$, the data well collapse onto a single curve, as shown in Fig. 4(b).

How to account for this universal relationship? For $\theta_{m}=0$, the whole RBC cell can be considered as an almost $\mathrm{OB}$ one, which is characterized by $\mathrm{Ra}$ and the corresponding critical Rayleigh number $\mathrm{Ra}_{c, 0}$ for the onset of convection in this cell. For $\theta_{m}>0$, only the lower layer can be treated as an OB cell. The temperature at this layer's upper surface is $T_{m}$ and the corresponding Rayleigh number and critical Rayleigh number are $\mathrm{Ra}_{\ell}$ and $\mathrm{Ra}_{c}$, respectively. From 2D OB DNS with $\mathrm{Pr}=10$ [30] we know that in the considered $\mathrm{Ra}$ range, $\mathrm{Nu}$ scales as $\left(\mathrm{Ra} / \mathrm{Ra}_{c, 0}\right)^{\gamma}$ with $\gamma \approx 0.27$. Therefore $\mathrm{Nu}\left(\theta_{m}\right) / \mathrm{Nu}(0)=\left(\mathrm{Ra}_{\ell} / \mathrm{Ra}_{c}\right)^{\gamma}\left(\mathrm{Ra}_{c, 0} / \mathrm{Ra}\right)^{\gamma}=\left(\mathrm{Ra}_{\ell} / \mathrm{Ra}\right)^{\gamma}\left(\mathrm{Ra}_{c, 0} / \mathrm{Ra}_{c}\right)^{\gamma}$. Due to the reduced height and the temperature drop in the lower layer compared to the whole RBC cell [by almost $\left(1-\theta_{m}\right)$, as a first approximation], the value of $\mathrm{Ra}_{\ell} / \mathrm{Ra}$ can be approximated as $\left(1-\theta_{m}\right)^{4}$. The critical Rayleigh numbers for the onset of convection can be approximated as $\operatorname{Ra}_{c} \sim\left[1+\left(1-\theta_{m}\right)^{2} / \Gamma^{2}\right]^{2}$ and $\mathrm{Ra}_{c, 0} \sim\left[1+1 / \Gamma^{2}\right]^{2}$ (see more information in Appendix $\mathrm{B}$ ), which altogether lead to

$$
\frac{\mathrm{Nu}\left(\theta_{m}\right)}{\mathrm{Nu}(0)} \approx\left[\frac{\left(1+\Gamma^{2}\right)\left(1-\theta_{m}\right)^{2}}{\Gamma^{2}+\left(1-\theta_{m}\right)^{2}}\right]^{2 \gamma}=\left[\frac{5\left(1-\theta_{m}\right)^{2}}{4+\left(1-\theta_{m}\right)^{2}}\right]^{0.54} \text {. }
$$



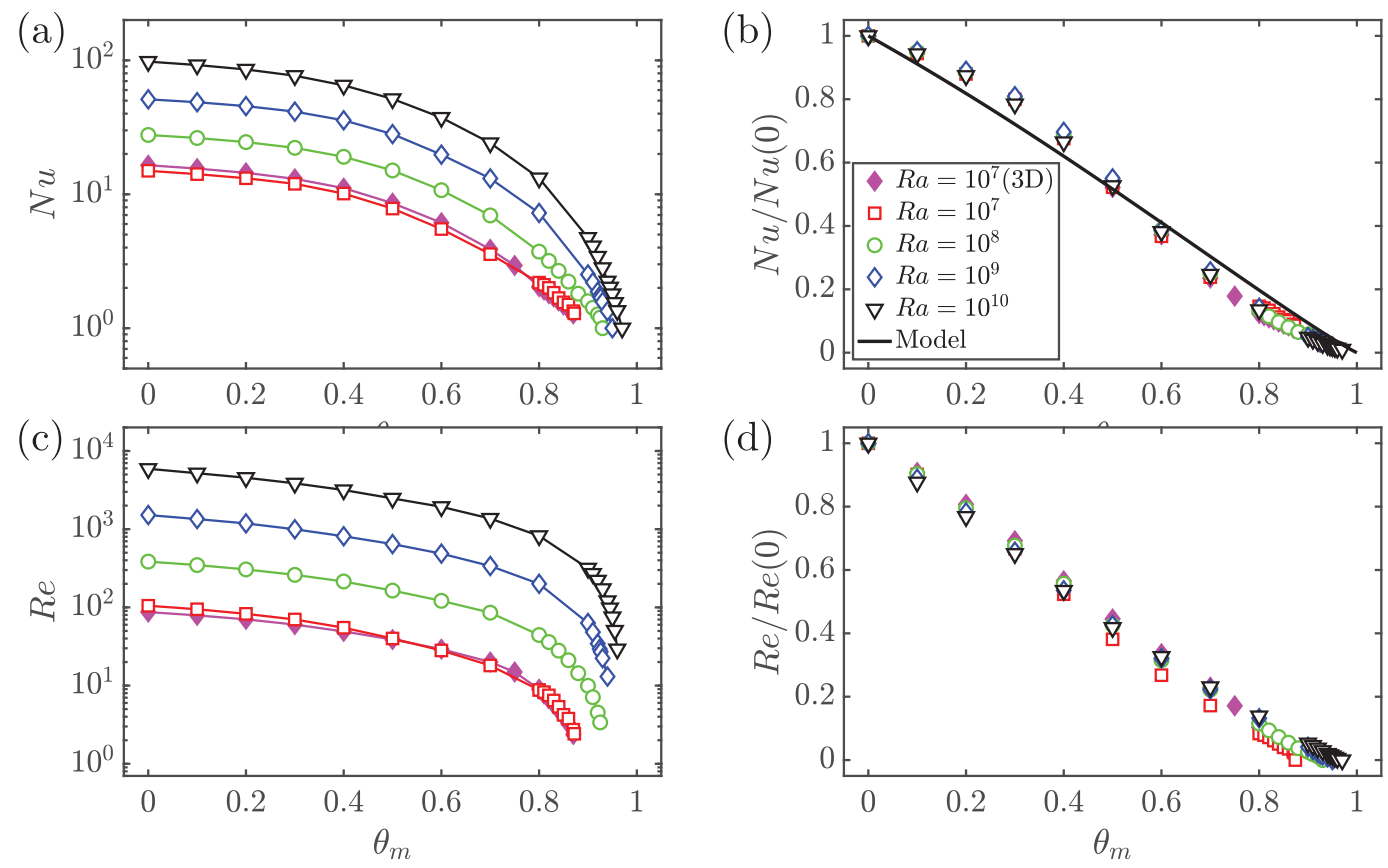

FIG. 4. Absolute and normalized Nusselt and Reynolds numbers as function of the density inversion parameter $\theta_{m}$ for different Ra: (a) absolute Nusselt number $\mathrm{Nu}$, (b) normalized Nusselt number $\mathrm{Nu}\left(\theta_{m}\right) / \mathrm{Nu}(0)$, (c) absolute Reynolds number $\operatorname{Re}$, (d) normalized Reynolds number $\operatorname{Re}\left(\theta_{m}\right) / \operatorname{Re}(0)$. Normalization was carried out using the corresponding values for $\theta_{m}=0$. The black line in panel (b) shows the theoretical model, Eq. (6), and the lines in panels (a) and (c) are used to guide the eye. Panels (a), (c), and (d) share the same legend as that in panel (b).

This prediction is consistent with the general trend of the normalized Nusselt number, as can be seen in Fig. 4(b).

Figure 4(c) shows the global Reynolds number as function of $\theta_{m}$ for both the 2D and 3D cases for various $\mathrm{Ra}$. It is seen that Re monotonically decreases with increasing $\theta_{m}$. The normalized Reynolds number $\operatorname{Re}\left(\theta_{m}\right) / \operatorname{Re}(0)$ also shows a universal dependence on $\theta_{m}$ (Fig. 4d), just as $\mathrm{Nu}\left(\theta_{m}\right) / \mathrm{Nu}(0)$ shown in Fig. 4(b).

Finally, we address the (rare) transitions between different flow states for large $\theta_{m}$. It was found that the Reynolds number ratio $\mathrm{Re}_{z} / \mathrm{Re}_{x}$ can well differentiate different convection roll states [30,31]. Here $\operatorname{Re}_{z}=\left\langle w^{2}\right\rangle_{V, t}^{1 / 2} H / v$ is the volume averaged vertical Reynolds number and $\operatorname{Re}_{x}=\left\langle u^{2}\right\rangle_{V, t}^{1 / 2} H / v$ the horizontal one. For $\mathrm{Ra}=10^{10}$, Fig. 5(a) shows that the statistically stable two-roll state, as shown in Fig. 1(b), can be found for up to $\theta_{m}=0.93$. However, once $\theta_{m}$ increases to $\theta_{m}=0.945$, the two-roll state is not sustained all the time and rare transitions between two-roll and four-roll states occur, which can be seen in the rare jumps of the Reynolds number ratio $\operatorname{Re}_{z} / \operatorname{Re}_{x}$ in Fig. 5(b). The temperature fields for the two-roll and four-roll states are shown in Figs. 5(d) and 5(e), respectively. At even larger $\theta_{m}=0.955$, constant jumps between different states are observed, as displayed in Fig. 5(c). The instantaneous temperature fields at two instants are shown in Figs. 5(f) and 5(g). It can be seen that and the convection rolls are now not well organised. When $\theta_{m}$ reaches 0.97 , the flow enters into the conduction state without any fluid motions.

Rare transitions between different turbulent states have been reported for many different flows [43-46]. Similar phenomena are also important in many geophysical flows $[47,48]$. Here we have thus found another example of such rare transition events in turbulent flows, which were not identified for OB cases [30]. Similar to prior studies [45,46], such a phenomenon can be viewed as flow mode competition between different states: for not very large $\theta_{m}$, e.g., $\theta_{m}=0.93$, the effective 

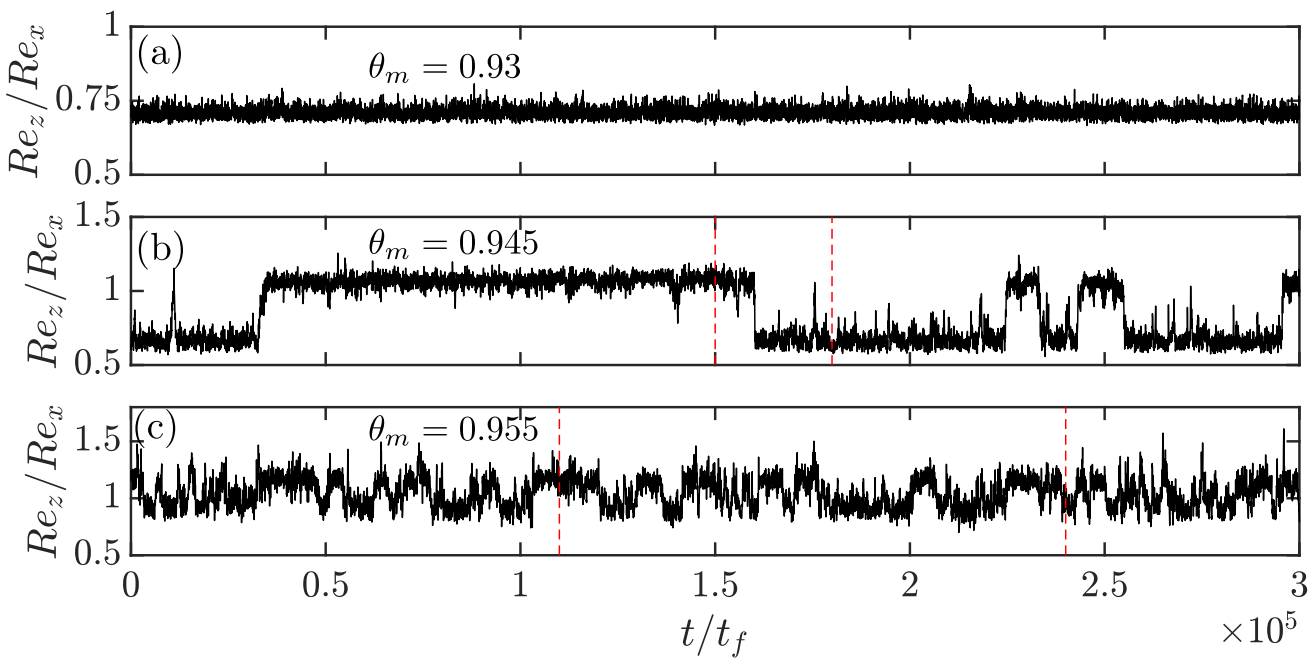

(d) $\theta_{m}=0.945, t / t_{f}=1.5 \times 10^{5}$

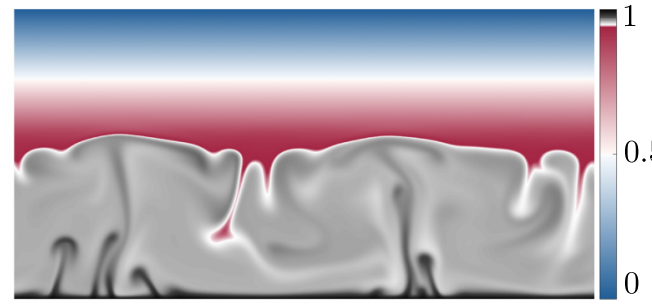

(f) $\theta_{m}=0.955, t / t_{f}=1.1 \times 10^{5}$

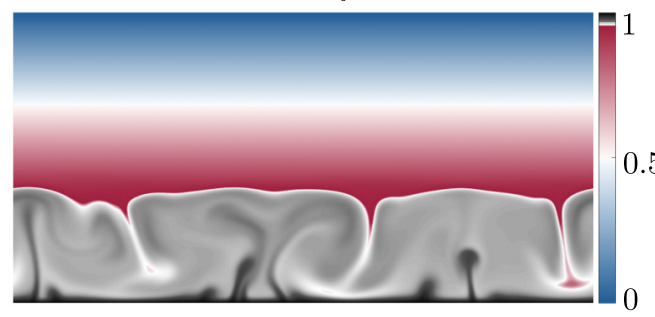

(e) $\theta_{m}=0.945, t / t_{f}=1.8 \times 10^{5}$

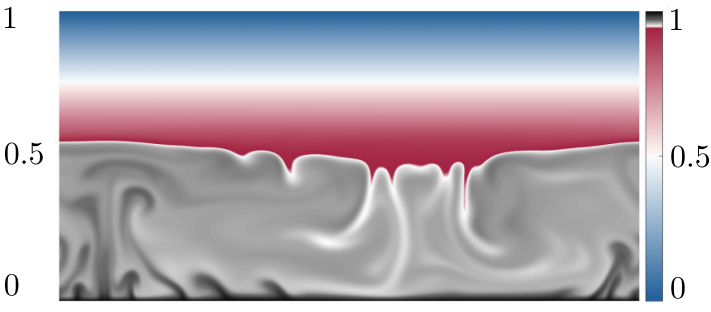

(g) $\theta_{m}=0.955, t / t_{f}=2.4 \times 10^{5}$

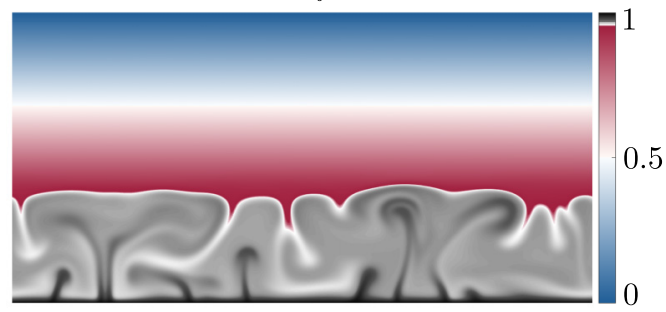

FIG. 5. Time evolution of the Reynolds number ratio $\operatorname{Re}_{z} / \operatorname{Re}_{x}$ for $\mathrm{Ra}=10^{10}$ with (a) $\theta_{m}=0.93$, (b) $\theta_{m}=$ 0.945, and (c) $\theta_{m}=0.955$. Instantaneous temperature fields for $\theta_{m}=0.945$ and $\theta_{m}=0.955$ at different times denoted by dashed lines in panels (b) and (c): (d) $\theta_{m}=0.945, t / t_{f}=1.5 \times 10^{5}$, (e) $\theta_{m}=0.945, t / t_{f}=1.8 \times$ $10^{5}$, (f) $\theta_{m}=0.955, t / t_{f}=1.1 \times 10^{5}$, and (g) $\theta_{m}=0.955, t / t_{f}=2.4 \times 10^{5}$.

aspect ratio of the convection region is close to $\Gamma=2$, therefore, it supports only a two-roll state. At large $\theta_{m}$ close to $\theta_{m}=1$, the effective aspect ratio becomes much larger than 1 , and therefore it can support more convection rolls [30]. In between, the different convection roll states may have comparable strength, and the competition of these states leads to the rare transitions between them.

\section{SUMMARY}

In conclusion, based on our DNS of turbulent penetrative RBC in cold water, where the density anomaly temperature is achieved in the cell's bulk, we have shown that the main response parameters, namely, the mean central temperature $\theta_{c}$ and the normalized Nusselt number, are universally determined by the density inversion parameter $\theta_{m}$. For $\theta_{m}=0$, the convective system 
is similar to that in the Oberbeck-Boussinesq case. However, for $\theta_{m}$ approaching 1, the convection vanishes at a certain $\theta_{m}=\theta_{m, c}$ as soon as the Rayleigh number of the lower layer achieves the critical Rayleigh number $\mathrm{Ra}_{c}$ for the onset of convection. We have demonstrated that $\mathrm{Ra}_{c}$ and hence $\theta_{m, c}$ can be excellently predicted by a linear stability analysis. We have further shown that in the range $0 \leqslant \theta_{m} \lesssim \theta_{m, c}$, the central temperature $\theta_{c}\left(\theta_{m}\right)$ is independent of Ra and can be excellently predicted by our model. Furthermore, we proposed a theoretical model for the normalized heat transport.

\section{ACKNOWLEDGMENTS}

R. Verzicco is gratefully acknowledged for continuous collaborations and discussions. We also acknowledge the Twente Max-Planck Center and the Deutsche Forschungsgemeinschaft (Priority Programme SPP 1881 "Turbulent Superstructures" and Grant No. Sh405/10). The simulations were carried out on the national e-infrastructure of SURFsara, a subsidiary of SURF cooperation, the collaborative ICT organization for Dutch education and research. Q.W. acknowledges financial support from the China Scholarship Council (CSC) and the Natural Science Foundation of China (NSFC) under Grant No. 11621202.

\section{APPENDIX A: DETAILS OF DIRECT NUMERICAL SIMULATIONS}

This section gives details about the 2D (Table I) and 3D (Table II) simulations.

TABLE I. Details of the 2D simulations. The columns from left to right indicate the Rayleigh number $\mathrm{Ra}$, the Prandtl number Pr, the density inversion parameter $\theta_{m}$, aspect ratio $\Gamma$, grid resolutions $N_{x} \times N_{z}$, Nusselt number $\mathrm{Nu}$, Reynolds number Re, central temperature $\theta_{c}$, and the time $t_{\text {avg }}$ used to average $\mathrm{Nu}$ and Re. "Steady" means that the flow is in a steady state, and "conductive" means that the flow is in a conductive state without any fluid motions.

\begin{tabular}{lcccccrcc}
\hline \hline $\mathrm{Ra}$ & $\operatorname{Pr}$ & $\theta_{m}$ & $\Gamma$ & $N_{x} \times N_{z}$ & $\mathrm{Nu}$ & $\mathrm{Re}$ & $\theta_{c}$ & $t_{\text {avg }}$ \\
\hline $10^{7}$ & 11.57 & 0 & 2 & $256 \times 128$ & 14.97 & 104.96 & 0.5244 & 4000 \\
$10^{7}$ & 11.57 & 0.1 & 2 & $256 \times 128$ & 14.14 & 94.63 & 0.5297 & 4000 \\
$10^{7}$ & 11.57 & 0.2 & 2 & $256 \times 128$ & 13.17 & 82.67 & 0.5359 & 4000 \\
$10^{7}$ & 11.57 & 0.3 & 2 & $256 \times 128$ & 11.97 & 70.04 & 0.5532 & 4000 \\
$10^{7}$ & 11.57 & 0.4 & 2 & $256 \times 128$ & 10.12 & 54.95 & 0.5767 & 4000 \\
$10^{7}$ & 11.57 & 0.5 & 2 & $256 \times 128$ & 7.82 & 40.01 & 0.6311 & 4000 \\
$10^{7}$ & 11.57 & 0.6 & 2 & $256 \times 128$ & 5.50 & 28.12 & 0.7027 & 4000 \\
$10^{7}$ & 11.57 & 0.7 & 2 & $256 \times 128$ & 3.57 & 18.06 & 0.7718 & 4000 \\
$10^{7}$ & 11.57 & 0.8 & 2 & $256 \times 128$ & 2.19 & 8.78 & 0.8481 & 2000 \\
$10^{7}$ & 11.57 & 0.81 & 2 & $256 \times 128$ & 2.11 & 8.25 & 0.8502 & 10000 \\
$10^{7}$ & 11.57 & 0.82 & 2 & $256 \times 128$ & 2.00 & 7.50 & 0.8524 & Steady \\
$10^{7}$ & 11.57 & 0.83 & 2 & $256 \times 128$ & 1.84 & 6.45 & 0.8492 & Steady \\
$10^{7}$ & 11.57 & 0.84 & 2 & $256 \times 128$ & 1.68 & 5.36 & 0.8282 & Steady \\
$10^{7}$ & 11.57 & 0.85 & 2 & $256 \times 128$ & 1.56 & 4.23 & 0.7866 & 25000 \\
$10^{7}$ & 11.57 & 0.86 & 2 & $256 \times 128$ & 1.50 & 3.78 & 0.7578 & Steady \\
$10^{7}$ & 11.57 & 0.865 & 2 & $256 \times 128$ & 1.43 & 3.30 & 0.7116 & Steady \\
$10^{7}$ & 11.57 & 0.87 & 2 & $256 \times 128$ & 1.34 & 2.73 & 0.6690 & Steady \\
$10^{7}$ & 11.57 & 0.872 & 2 & $256 \times 128$ & 1.29 & 2.42 & 0.6448 & Steady \\
$10^{7}$ & 11.57 & 0.873 & 2 & $256 \times 128$ & 1.25 & 2.15 & 0.6225 & Steady \\
$10^{7}$ & 11.57 & 0.8735 & 2 & $256 \times 128$ & & & & Conductive \\
$10^{8}$ & 11.57 & 0 & 2 & $512 \times 256$ & 27.72 & 384.92 & 0.5272 & 4000 \\
$10^{8}$ & 11.57 & 0.1 & 2 & $512 \times 256$ & 26.32 & 347.48 & 0.5326 & 4000 \\
$10^{8}$ & 11.57 & 0.2 & 2 & $512 \times 256$ & 24.52 & 305.80 & 0.5429 & 4000 \\
$10^{8}$ & 11.57 & 0.3 & 2 & $512 \times 256$ & 22.22 & 260.52 & 0.5569 & 4000 \\
\hline
\end{tabular}


TABLE I. (Continued.)

\begin{tabular}{|c|c|c|c|c|c|c|c|c|}
\hline $\mathrm{Ra}$ & $\operatorname{Pr}$ & $\theta_{m}$ & $\Gamma$ & $N_{x} \times N_{z}$ & $\mathrm{Nu}$ & $\mathrm{Re}$ & $\theta_{c}$ & $t_{\text {avg }}$ \\
\hline $10^{7}$ & 11.57 & 0.4 & 2 & $512 \times 256$ & 19.05 & 214.35 & 0.5892 & 4000 \\
\hline $10^{7}$ & 11.57 & 0.5 & 2 & $512 \times 256$ & 15.06 & 163.90 & 0.6347 & 4000 \\
\hline $10^{7}$ & 11.57 & 0.6 & 2 & $512 \times 256$ & 10.73 & 121.32 & 0.6987 & 4000 \\
\hline $10^{7}$ & 11.57 & 0.7 & 2 & $512 \times 256$ & 6.95 & 85.55 & 0.7743 & 3000 \\
\hline $10^{7}$ & 11.57 & 0.8 & 2 & $512 \times 256$ & 3.73 & 44.32 & 0.8514 & 3000 \\
\hline $10^{7}$ & 11.57 & 0.82 & 2 & $512 \times 256$ & 3.18 & 36.04 & 0.8665 & 15000 \\
\hline $10^{7}$ & 11.57 & 0.84 & 2 & $512 \times 256$ & 2.68 & 28.00 & 0.8797 & 15000 \\
\hline $10^{7}$ & 11.57 & 0.86 & 2 & $512 \times 256$ & 2.23 & 21.09 & 0.8921 & 35000 \\
\hline $10^{7}$ & 11.57 & 0.88 & 2 & $512 \times 256$ & 1.81 & 14.40 & 0.8774 & 35000 \\
\hline $10^{7}$ & 11.57 & 0.9 & 2 & $512 \times 256$ & 1.59 & 9.95 & 0.8000 & 10000 \\
\hline $10^{7}$ & 11.57 & 0.91 & 2 & $256 \times 128$ & 1.42 & 7.10 & 0.7078 & Steady \\
\hline $10^{7}$ & 11.57 & 0.92 & 2 & $256 \times 128$ & 1.27 & 4.52 & 0.6352 & Steady \\
\hline $10^{7}$ & 11.57 & 0.925 & 2 & $256 \times 128$ & 1.20 & 3.38 & 0.6025 & Steady \\
\hline $10^{7}$ & 11.57 & 0.93 & 2 & $256 \times 128$ & & & & Conductive \\
\hline $10^{9}$ & 11.57 & 0 & 2 & $1024 \times 512$ & 51.16 & 1515.45 & 0.5216 & 2000 \\
\hline $10^{9}$ & 11.57 & 0.1 & 2 & $1024 \times 512$ & 48.68 & 1346.21 & 0.5284 & 2000 \\
\hline $10^{9}$ & 11.57 & 0.2 & 2 & $1024 \times 512$ & 45.52 & 1184.81 & 0.5377 & 2000 \\
\hline $10^{9}$ & 11.57 & 0.3 & 2 & $1024 \times 512$ & 41.42 & 996.96 & 0.5565 & 2000 \\
\hline $10^{9}$ & 11.57 & 0.4 & 2 & $1024 \times 512$ & 35.66 & 811.87 & 0.5909 & 2000 \\
\hline $10^{9}$ & 11.57 & 0.5 & 2 & $1024 \times 512$ & 28.14 & 644.66 & 0.6401 & 2000 \\
\hline $10^{9}$ & 11.57 & 0.6 & 2 & $1024 \times 512$ & 19.76 & 486.75 & 0.6954 & 2000 \\
\hline $10^{9}$ & 11.57 & 0.7 & 2 & $1024 \times 512$ & 13.13 & 338.08 & 0.7717 & 2000 \\
\hline $10^{9}$ & 11.57 & 0.8 & 2 & $1024 \times 512$ & 7.25 & 199.97 & 0.8480 & 6000 \\
\hline $10^{9}$ & 11.57 & 0.9 & 2 & $1024 \times 512$ & 2.52 & 63.07 & 0.9242 & 4000 \\
\hline $10^{9}$ & 11.57 & 0.91 & 2 & $512 \times 256$ & 2.20 & 48.26 & 0.9326 & 30000 \\
\hline $10^{9}$ & 11.57 & 0.92 & 2 & $512 \times 256$ & 1.87 & 34.34 & 0.9173 & 20000 \\
\hline $10^{9}$ & 11.57 & 0.924 & 2 & $512 \times 256$ & 1.74 & 29.38 & 0.8765 & 10000 \\
\hline $10^{9}$ & 11.57 & 0.926 & 2 & $512 \times 256$ & 1.68 & 26.98 & 0.8443 & 10000 \\
\hline $10^{9}$ & 11.57 & 0.93 & 2 & $512 \times 256$ & 1.57 & 22.36 & 0.7859 & 20000 \\
\hline $10^{9}$ & 11.57 & 0.94 & 2 & $512 \times 256$ & 1.34 & 13.04 & 0.6722 & 10000 \\
\hline $10^{9}$ & 11.57 & 0.945 & 2 & $512 \times 256$ & 1.31 & 11.45 & 0.6544 & Steady \\
\hline $10^{9}$ & 11.57 & 0.95 & 2 & $512 \times 256$ & & & & Conductive \\
\hline $10^{10}$ & 11.57 & 0 & 2 & $2048 \times 1024$ & 97.84 & 5919.68 & 0.5168 & 1000 \\
\hline $10^{10}$ & 11.57 & 0.1 & 2 & $2048 \times 1024$ & 92.26 & 5191.37 & 0.5250 & 1000 \\
\hline $10^{10}$ & 11.57 & 0.2 & 2 & $2048 \times 1024$ & 85.51 & 4546.96 & 0.5336 & 2000 \\
\hline $10^{10}$ & 11.57 & 0.3 & 2 & $2048 \times 1024$ & 76.76 & 3855.20 & 0.5524 & 2000 \\
\hline $10^{10}$ & 11.57 & 0.4 & 2 & $2048 \times 1024$ & 65.06 & 3166.88 & 0.5851 & 2000 \\
\hline $10^{10}$ & 11.57 & 0.5 & 2 & $2048 \times 1024$ & 51.53 & 2473.53 & 0.6302 & 2000 \\
\hline $10^{10}$ & 11.57 & 0.6 & 2 & $2048 \times 1024$ & 37.36 & 1931.61 & 0.6905 & 4000 \\
\hline $10^{10}$ & 11.57 & 0.7 & 2 & $2048 \times 1024$ & 24.15 & 1366.69 & 0.7676 & 3000 \\
\hline $10^{10}$ & 11.57 & 0.8 & 2 & $2048 \times 1024$ & 13.20 & 821.26 & 0.8466 & 8000 \\
\hline $10^{10}$ & 11.57 & 0.9 & 2 & $2048 \times 1024$ & 4.74 & 315.42 & 0.9242 & 2000 \\
\hline $10^{10}$ & 11.57 & 0.91 & 2 & $512 \times 256$ & 4.10 & 269.86 & 0.9317 & 30000 \\
\hline $10^{10}$ & 11.57 & 0.92 & 2 & $512 \times 256$ & 3.43 & 221.30 & 0.9391 & 30000 \\
\hline $10^{10}$ & 11.57 & 0.93 & 2 & $512 \times 256$ & 2.82 & 172.40 & 0.9468 & 30000 \\
\hline $10^{10}$ & 11.57 & 0.94 & 2 & $512 \times 256$ & 2.24 & 120.80 & 0.9548 & 10000 \\
\hline $10^{10}$ & 11.57 & 0.945 & 2 & $512 \times 256$ & 2.02 & 97.73 & 0.9522 & 70000 \\
\hline $10^{10}$ & 11.57 & 0.95 & 2 & $512 \times 256$ & 1.80 & 75.19 & 0.9003 & 30000 \\
\hline $10^{10}$ & 11.57 & 0.955 & 2 & $512 \times 256$ & 1.55 & 50.57 & 0.7832 & 5000 \\
\hline $10^{10}$ & 11.57 & 0.96 & 2 & $512 \times 256$ & 1.35 & 29.21 & 0.6743 & 30000 \\
\hline $10^{10}$ & 11.57 & 0.965 & 2 & $512 \times 256$ & 1.20 & 15.96 & 0.6037 & 10000 \\
\hline $10^{10}$ & 11.57 & 0.97 & 2 & $512 \times 256$ & & & & Conductive \\
\hline
\end{tabular}


TABLE II. Details of the 3D simulations. The columns from left to right indicate the Rayleigh number Ra, the Prandtl number Pr, the density inversion parameter $\theta_{m}$, aspect ratio $\Gamma$, grid resolutions $N_{x} \times N_{y} \times N_{z}$, Nusselt number $\mathrm{Nu}$, Reynolds number Re, central temperature $\theta_{c}$, and the time $t_{\text {avg }}$ used to average Nu and Re. "Conductive" means that the flow is in a conductive state without any fluid motions.

\begin{tabular}{lcccccccc}
\hline \hline $\operatorname{Ra}$ & $\operatorname{Pr}$ & $\theta_{m}$ & $\Gamma$ & $N_{x} \times N_{y} \times N_{z}$ & $\mathrm{Nu}$ & $\mathrm{Re}$ & $\theta_{c}$ & $t_{\text {avg }}$ \\
\hline $10^{7}$ & 11.57 & 0 & 4 & $512 \times 512 \times 128$ & 16.47 & 87.07 & 0.5221 & 600 \\
$10^{7}$ & 11.57 & 0.1 & 4 & $512 \times 512 \times 128$ & 15.56 & 79.03 & 0.5276 & 500 \\
$10^{7}$ & 11.57 & 0.2 & 4 & $512 \times 512 \times 128$ & 14.46 & 70.27 & 0.5369 & 500 \\
$10^{7}$ & 11.57 & 0.3 & 4 & $512 \times 512 \times 128$ & 13.04 & 60.25 & 0.5502 & 500 \\
$10^{7}$ & 11.57 & 0.4 & 4 & $512 \times 512 \times 128$ & 11.12 & 49.26 & 0.5808 & 1200 \\
$10^{7}$ & 11.57 & 0.5 & 4 & $512 \times 512 \times 128$ & 8.59 & 38.78 & 0.6374 & 1500 \\
$10^{7}$ & 11.57 & 0.6 & 4 & $512 \times 512 \times 128$ & 6.13 & 29.22 & 0.7108 & 800 \\
$10^{7}$ & 11.57 & 0.7 & 4 & $256 \times 256 \times 64$ & 3.88 & 20.02 & 0.7822 & 1000 \\
$10^{7}$ & 11.57 & 0.75 & 4 & $256 \times 256 \times 64$ & 2.94 & 14.91 & 0.8188 & 3000 \\
$10^{7}$ & 11.57 & 0.8 & 4 & $256 \times 256 \times 64$ & 2.04 & 8.99 & 0.8316 & 1000 \\
$10^{7}$ & 11.57 & 0.81 & 4 & $256 \times 256 \times 64$ & 1.93 & 7.82 & 0.8252 & 5000 \\
$10^{7}$ & 11.57 & 0.82 & 4 & $256 \times 256 \times 64$ & 1.81 & 6.78 & 0.8215 & 5000 \\
$10^{7}$ & 11.57 & 0.83 & 4 & $256 \times 256 \times 64$ & 1.71 & 5.85 & 0.8172 & 5000 \\
$10^{7}$ & 11.57 & 0.84 & 4 & $256 \times 256 \times 64$ & 1.60 & 4.87 & 0.8003 & 5000 \\
$10^{7}$ & 11.57 & 0.85 & 4 & $256 \times 256 \times 64$ & 1.49 & 4.05 & 0.7638 & 5000 \\
$10^{7}$ & 11.57 & 0.86 & 4 & $256 \times 256 \times 64$ & 1.40 & 3.25 & 0.7111 & 5000 \\
$10^{7}$ & 11.57 & 0.87 & 4 & $256 \times 256 \times 64$ & 1.28 & 2.37 & 0.6513 & 5000 \\
$10^{7}$ & 11.57 & 0.88 & 4 & $256 \times 256 \times 64$ & & & & Conductive \\
\hline \hline
\end{tabular}

\section{APPENDIX B: LINEAR STABILITY ANALYSIS FOR RAYLEIGH-BÉNARD CONVECTION IN COLD WATER}

\section{Linearization of the buoyancy term}

To conduct linear stability analysis, we linearize the governing equations in a standard way with $\theta=\Theta+\theta^{\prime}$, where $\Theta=\Theta(z), 0 \leqslant \Theta \leqslant 1$, is the temperature profile in the steady case and $\theta^{\prime}$ is the deviation of the temperature from that in the steady case. The linearized buoyancy term follows from the following approximation (that holds for small values of $\theta^{\prime}$ ):

$$
\left|\theta-\theta_{m}\right|^{q}=\left|\theta^{\prime}+\Theta-\theta_{m}\right|^{q} \approx\left|\Theta-\theta_{m}\right|^{q}+\operatorname{sign}\left(\Theta-\theta_{m}\right) q \theta^{\prime}\left|\Theta-\theta_{m}\right|^{q-1}+\mathcal{O}\left(\theta^{\prime 2}\right) .
$$

TABLE III. Critical Rayleigh number for the onset of convection in an infinite layer of cold water, with no-slip top and bottom boundary conditions.

\begin{tabular}{cc}
\hline \hline$\theta_{m}$ & $\operatorname{Ra}_{c}$ \\
\hline 0.0 & $1.68 \times 10^{3}$ \\
0.1 & $2.06 \times 10^{3}$ \\
0.2 & $2.66 \times 10^{3}$ \\
0.3 & $3.82 \times 10^{3}$ \\
0.4 & $6.75 \times 10^{3}$ \\
0.5 & $1.68 \times 10^{4}$ \\
0.6 & $5.17 \times 10^{4}$ \\
0.7 & $2.08 \times 10^{5}$ \\
0.8 & $1.52 \times 10^{6}$ \\
0.9 & $4.47 \times 10^{7}$ \\
\hline
\end{tabular}


TABLE IV. Critical Rayleigh number for the onset of convection in an infinite layer of cold water, with no-slip bottom and free-slip top boundary conditions.

\begin{tabular}{cc}
\hline \hline$\theta_{m}$ & $\mathrm{Ra}_{c}$ \\
\hline 0.0 & $1.17 \times 10^{3}$ \\
0.1 & $1.46 \times 10^{3}$ \\
0.2 & $1.97 \times 10^{3}$ \\
0.3 & $3.05 \times 10^{3}$ \\
0.4 & $6.26 \times 10^{3}$ \\
0.5 & $1.71 \times 10^{4}$ \\
0.6 & $5.08 \times 10^{4}$ \\
0.7 & $2.07 \times 10^{5}$ \\
0.8 & $1.52 \times 10^{6}$ \\
0.9 & $4.48 \times 10^{7}$ \\
\hline \hline
\end{tabular}

TABLE V. Critical Rayleigh number for the onset of convection in a 2D domain with no-slip bottom and top BCs and periodic BCs in the horizontal direction. The fluid properties are as in cold water.

\begin{tabular}{cc}
\hline \hline$\theta_{m}$ & $\mathrm{Ra}_{c}$ \\
\hline 0.0 & $1.70 \times 10^{3}$ \\
0.1 & $2.15 \times 10^{3}$ \\
0.2 & $2.71 \times 10^{3}$ \\
0.3 & $3.83 \times 10^{3}$ \\
0.4 & $6.83 \times 10^{3}$ \\
0.5 & $1.89 \times 10^{4}$ \\
0.6 & $5.48 \times 10^{4}$ \\
0.7 & $2.07 \times 10^{5}$ \\
0.8 & $1.53 \times 10^{6}$ \\
0.9 & $3.92 \times 10^{7}$ \\
\hline \hline
\end{tabular}

TABLE VI. Critical Rayleigh number for the onset of convection in a 2D domain with no-slip bottom BC and free-slip top BC and periodic BCs in the horizontal direction. The fluid properties are as in cold water.

\begin{tabular}{cc}
\hline \hline$\theta_{m}$ & $\mathrm{Ra}_{c}$ \\
\hline 0.000 & $1.21 \times 10^{3}$ \\
0.100 & $1.52 \times 10^{3}$ \\
0.200 & $2.04 \times 10^{3}$ \\
0.300 & $3.12 \times 10^{3}$ \\
0.400 & $6.30 \times 10^{3}$ \\
0.500 & $1.89 \times 10^{4}$ \\
0.600 & $5.40 \times 10^{4}$ \\
0.700 & $2.07 \times 10^{5}$ \\
0.800 & $1.43 \times 10^{6}$ \\
0.850 & $6.22 \times 10^{6}$ \\
0.875 & $1.50 \times 10^{7}$ \\
0.900 & $3.92 \times 10^{7}$ \\
0.925 & $1.78 \times 10^{8}$ \\
0.950 & $1.30 \times 10^{9}$ \\
0.970 & $1.57 \times 10^{10}$ \\
\hline
\end{tabular}


TABLE VII. Critical Rayleigh number for the onset of convection in cold water for $\theta_{m}=0$ (the temperature at the top equals the temperature of the density anomaly), in a 2D domain with no-slip top and bottom BCs and periodic BCs at the side walls.

\begin{tabular}{cc}
\hline \hline$\Gamma$ & $\mathrm{Ra}_{c}$ \\
\hline 0.1 & $7.84 \times 10^{6}$ \\
0.2 & $6.37 \times 10^{5}$ \\
0.5 & $2.74 \times 10^{4}$ \\
1 & $3.72 \times 10^{3}$ \\
2 & $1.68 \times 10^{3}$ \\
5 & $1.77 \times 10^{3}$ \\
10 & $1.68 \times 10^{3}$ \\
\hline \hline
\end{tabular}

Therefore the buoyancy term in the linearized momentum equation equals $\operatorname{sgn}\left(\Theta-\theta_{m}\right) q \theta^{\prime} \mid \Theta-$ $\left.\theta_{m}\right|^{q-1} \overrightarrow{\boldsymbol{e}}_{z}$

In the case $\theta_{m}=0$, the buoyancy term can be $\operatorname{simplified}$ as $\operatorname{sgn}\left(\Theta-\theta_{m}\right) q \theta^{\prime}\left|\Theta-\theta_{m}\right|^{q-1}=$ $q \theta^{\prime} \Theta^{q-1}$, with $q=1.895$.

2. $\theta_{m}$-dependence of the critical Rayleigh number for the onset of convection in an infinite layer of cold water

Tables III and IV present the critical Rayleigh numbers for the case of no-slip BCs at both plates (Table III) and for the case where the bottom BC is no-slip and the top BC is free-slip (Table IV).

\section{3. $\theta_{m}$-dependence of the critical Rayleigh number for the onset of convection in a $2 \mathrm{D}$ domain of the aspect ratio $\Gamma=2$, for periodic BCs in horizontal direction}

Tables V and VI present the critical Rayleigh numbers for the onset of convection in a 2D domain, for periodic BCs in the horizontal direction and for the case of no-slip BCs at both plates (Table V) and the case where the bottom BC is no-slip and the top BC is free-slip (Table VI).

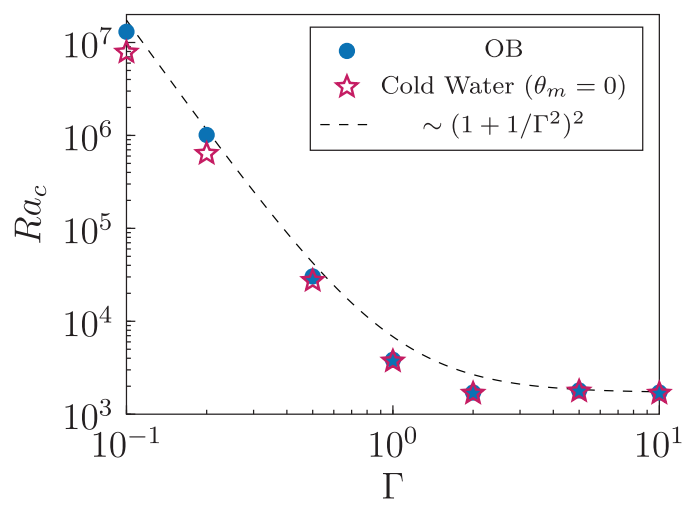

FIG. 6. Dependence of the critical Rayleigh number $\mathrm{Ra}_{c}$ for the onset of convection on aspect ratio $\Gamma$ in a 2D domain with no-slip BCs at the plates and periodic BCs at the side walls, for $\theta_{m}=0$. 


\section{Aspect ratio dependence of the critical Rayleigh number for the onset of convection, for $\boldsymbol{\theta}_{\boldsymbol{m}}=\mathbf{0}$}

Finally, in Table VII and Fig. 6 we present the dependence of the critical Rayleigh number on the aspect ratio $\Gamma$ of the 2D domain, for the case $\theta_{m}=0$.

[1] G. Ahlers, S. Grossmann, and D. Lohse, Heat transfer and large scale dynamics in turbulent RayleighBénard convection, Rev. Mod. Phys. 81, 503 (2009).

[2] D. Lohse and K.-Q. Xia, Small-scale properties of turbulent Rayleigh-Bénard convection, Annu. Rev. Fluid Mech. 42, 335 (2010).

[3] F. Chillà and J. Schumacher, New perspectives in turbulent Rayleigh-Bénard convection, Eur. Phys. J. E 35, 58 (2012).

[4] J. M. Dumore, H. J. Merk, and J. A. Prins, Heat transfer from water to ice by thermal convection, Nature (London) 172, 460 (1953).

[5] V. P. Carey, B. Gebhart, and J. C. Mollendorf, Buoyancy force reversals in vertical natural convection flows in cold water, J. Fluid Mech. 97, 279 (1980).

[6] V. P. Carey and B. Gebhart, Visualization of the flow adjacent to a vertical ice surface melting in cold pure water, J. Fluid Mech. 107, 37 (1981).

[7] Z. Wang, E. Calzavarini, C. Sun, and F. Toschi, How the growth of lake ice depends on the fluid dynamics underneath, Proc. Nat. Acad. Sci. U. S. A. 118, e2012870118 (2021).

[8] G. Veronis, Penetrative convection. Astrophys. J. 137, 641 (1963).

[9] S. Musman, Penetrative convection, J. Fluid Mech. 31, 343 (1968).

[10] B. Dintrans, A. Brandenburg, Å. Nordlund, and R. F. Stein, Spectrum and amplitudes of internal gravity waves excited by penetrative convection in solar-type stars, Astron. Astrophys. 438, 365 (2005).

[11] B. Buffett, Geomagnetic fluctuations reveal stable stratification at the top of the Earth's core, Nature (London) 507, 484 (2014).

[12] K. Zhang and G. Schubert, Penetrative convection and zonal flow on jupiter, Science 273, 941 (1996).

[13] B. Gebhart and J. C. Mollendorf, A new density relation for pure and saline water, Deep-Sea Res. 24, 831 (1977).

[14] D. R. Moore and N. O. Weiss, Nonlinear penetrative convection, J. Fluid Mech. 61, 553 (1973).

[15] R. W. Walden and G. Ahlers, Non-Boussinesq and penetrative convection in a cylindrical cell, J. Fluid Mech. 109, 89 (1981).

[16] E. Large and C. D. Andereck, Penetrative Rayleigh-Bénard convection in water near its maximum density point, Phys. Fluids 26, 094101 (2014).

[17] Z. Ding and J. Wu, Coherent heat transport in 2D penetrative Rayleigh-Bénard convection, J. Fluid Mech. 920, A48 (2021).

[18] D. Lecoanet, M. Le Bars, K. J. Burns, G. M. Vasil, B. P. Brown, E. Quataert, and J. S. Oishi, Numerical simulations of internal wave generation by convection in water, Phys. Rev. E 91, 063016 (2015).

[19] Q. Wang, Q. Zhou, Z.-H. Wan, and D.-J. Sun, Penetrative turbulent Rayleigh-Bénard convection in two and three dimensions, J. Fluid Mech. 870, 718 (2019).

[20] P. Léard, B. Favier, P. Le Gal, and M. Le Bars, Coupled convection and internal gravity waves excited in water around its density maximum at $4{ }^{\circ} \mathrm{C}$, Phys. Rev. Fluids 5, 024801 (2020).

[21] L.-A. Couston, D. Lecoanet, B. Favier, and M. Le Bars, Dynamics of mixed convective-stably-stratified fluids, Phys. Rev. Fluids 2, 094804 (2017).

[22] L.-A. Couston, D. Lecoanet, B. Favier, and M. Le Bars, Order Out of Chaos: Slowly Reversing Mean Flows Emerge from Turbulently Generated Internal Waves, Phys. Rev. Lett. 120, 244505 (2018).

[23] L.-A. Couston, D. Lecoanet, B. Favier, and M. Le Bars, The energy flux spectrum of internal waves generated by turbulent convection, J. Fluid Mech. 854, R3 (2018).

[24] S. Toppaladoddi and J. S. Wettlaufer, Penetrative convection at high Rayleigh numbers, Phys. Rev. Fluids 3, 043501 (2018). 
[25] L.-A. Couston, D. Lecoanet, B. Favier, and M. Le Bars, Shape and size of large-scale vortices: A generic fluid pattern in geophysical fluid dynamics, Phys. Rev. Research 2, 023143 (2020).

[26] L.-A. Couston and M. Siegert, Dynamic flows create potentially habitable conditions in antarctic subglacial lakes, Sci. Adv. 7, eabc3972 (2021).

[27] L.-A. Couston, Turbulent convection in subglacial lakes, J. Fluid Mech. 915, A31 (2021).

[28] R. Verzicco and P. Orlandi, A finite-difference scheme for three-dimensional incompressible flows in cylindrical coordinates, J. Comput. Phys. 123, 402 (1996).

[29] E. P. van der Poel, R. Ostilla-Mónico, J. Donners, and R. Verzicco, A pencil distributed finite difference code for strongly turbulent wall-bounded flows, Comput. Fluids 116, 10 (2015).

[30] Q. Wang, R. Verzicco, D. Lohse, and O. Shishkina, Multiple States in Turbulent Large-Aspect Ratio Thermal Convection: What Determines the Number of Convection Rolls? Phys. Rev. Lett. 125, 074501 (2020).

[31] Q. Wang, K.-L. Chong, R. J. A. M. Stevens, R. Verzicco, and D. Lohse, From zonal flow to convection rolls in Rayleigh-Bénard convection with free-slip plates, J. Fluid. Mech. 905, A21 (2020).

[32] O. Shishkina, R. J. A. M. Stevens, S. Grossmann, and D. Lohse, Boundary layer structure in turbulent thermal convection and its consequences for the required numerical resolution, New J. Phys. 12, 075022 (2010).

[33] D. Goluskin and E. P. van der Poel, Penetrative internally heated convection in two and three dimensions, J. Fluid Mech. 791, R6 (2016).

[34] D. Goluskin, Internally Heated Convection and Rayleigh-Bénard Convection (Springer, Berlin, 2016).

[35] Q. Wang, O. Shishkina, and D. Lohse, Scaling in internally heated convection: A unifying theory, Geophys. Res. Lett. 48, e2020GL091198 (2021).

[36] O. Shishkina, S. Grossmann, and D. Lohse, Heat and momentum transport scalings in horizontal convection, Geophys. Res. Lett. 43, 1219 (2016).

[37] O. Shishkina and S. Wagner, Prandtl-Number Dependence of Heat Transport in Laminar Horizontal Convection, Phys. Rev. Lett. 116, 024302 (2016).

[38] P. Reiter and O. Shishkina, Classical and symmetrical horizontal convection: Detaching plumes and oscillations, J. Fluid Mech. 892, R1 (2020).

[39] D. P. M. Van Gils, S. Huisman, G.-W. Bruggert, C. Sun, and D. Lohse, Torque Scaling in Turbulent Taylor-Couette Flow with Co-And Counterrotating Cylinders, Phys. Rev. Lett. 106, 024502 (2011).

[40] R. Ostilla-Mónico, R. J. A. M Stevens, S. Grossmann, R. Verzicco, and D. Lohse, Optimal Taylor-Couette flow: Direct numerical simulations, J. Fluid Mech. 719, 14 (2013).

[41] R. Ostilla-Mónico, E. P. van der Poel, R. Verzicco, S. Grossmann, and D. Lohse, Exploring the phase diagram of fully turbulent Taylor-Couette flow, J. Fluid Mech. 761, 1 (2014).

[42] E. P. van der Poel, R. J. A. M. Stevens, and D. Lohse, Comparison between two-and three-dimensional Rayleigh-Bénard convection, J. Fluid Mech. 736, 177 (2013).

[43] K. Sugiyama, R. Ni, R. J. A. M. Stevens, T. S. Chan, S.-Q. Zhou, H.-D. Xi, C. Sun, S. Grossmann, K.-Q. Xia, and D. Lohse, Flow Reversals in Thermally Driven Turbulence, Phys. Rev. Lett. 105, 034503 (2010).

[44] E. P. van der Poel, R. J. A. M Stevens, and D. Lohse, Connecting flow structures and heat flux in turbulent Rayleigh-Bénard convection, Phys. Rev. E 84, 045303(R) (2011).

[45] Y.-C. Xie, G.-Y. Ding, and K.-Q. Xia, Flow Topology Transition via Global Bifurcation in Thermally Driven Turbulence, Phys. Rev. Lett. 120, 214501 (2018).

[46] Q. Wang, Z.-H. Wan, R. Yan, and D.-J. Sun, Multiple states and heat transfer in two-dimensional tilted convection with large aspect ratios, Phys. Rev. Fluids 3, 113503 (2018).

[47] G. A. Glatzmaier and P. H. Roberts, A three-dimensional self-consistent computer simulation of a geomagnetic field reversal, Nature (London) 377, 203 (1995).

[48] E. R. Weeks, Y. Tian, J. S. Urbach, K. Ide, H. L. Swinney, and M. Ghil, Transitions between blocked and zonal flows in a rotating annulus with topography, Science 278, 1598 (1997). 